\section{Screening for immunogenic cell death}

\section{By Kai-Jye Lou, Staff Writer}

French researchers have used a fluorescence-based screening platform to identify small molecules that induced immunogenic cell death in mouse tumors. ${ }^{1}$ Next, the group plans to test the best hits in a Phase I/ II trial to treat locally invasive head and neck cancers in combination with chemotherapy.

In 2005, Guido Kroemer and colleagues first showed that the anthracycline chemotherapeutic doxorubicin could induce immunemediated death of cancer cells in addition to the known process of cell death through DNA damage. ${ }^{2}$ The immunogenic process was later shown to be mediated by multiple factors involved in apoptosis and autophagy. ${ }^{3-6}$

Based on those mechanistic findings, Kroemer and colleagues decided to design an automated epifluorescence microscopy-based screening platform that could detect several hallmarks of immunogenic cell death and thus be used to identify new compounds that trigger this process in a human osteosarcoma cell line.

First, the researchers validated the new screening platform using a library of 120 marketed cancer therapies. Based on the readouts, the most potent compounds included several known inducers of immunogenic cell death, including doxorubicin, daunorubicin and mitoxantrone. Two other top hits were drugs known to have immunogenic side effects: vincristine and vinorelbine.

Next, the researchers used the platform to screen for new inducers of immunogenic cell death. Of the top 10 hits, 4 were small molecule cardiac glycosides, including digoxin and digitoxin, which are marketed to reduce arrhythmias in patients with heart failure.

The researchers then tested digoxin in vivo as it is the more commonly used drug. In mice, digoxin stimulated immunogenic cancer cell death and elicited a protective antitumor immune response.

Finally, the researchers did a retrospective analysis of patients with various carcinomas receiving chemotherapy and found that those also on digoxin had greater survival rates than patients not on digoxin.

The findings were published in Science Translational Medicine. Kroemer is a professor in the faculty of medicine at the University Paris Descartes, director of the apoptosis, cancer and immunity unit at Institut National de la Santé et de la Recherche Médicale (INSERM), hospital practitioner at Georges Pompidou European Hospital and director of the Metabolomics facility at the Gustave Roussy Institute.
"The most striking result from this study was that the group was also able to correlate their preclinical data with retrospective patient data," said Ignacio Melero, a professor of immunology and senior consultant at the University Clinic of Navarra at the University of Navarra. "Because the chemotherapies and cardiac glycosides assessed in the retrospective analysis have already seen decades of patient use and have established safety profiles, it should be easy to test the combinations in the clinic."

The use of cardiac glycosides in the cancer setting had been suggested by past research, though the mechanisms underlying their anticancer effects in those studies were unclear. ${ }^{7}$

"The greatest potential of this platform is in identifying and evaluating smart combination therapeutic regimens because it allows one to screen for compounds that are immunostimulatory but not necessarily very cytotoxic. For example, one could envision combining a very potent, very cytotoxic agent with a less cytotoxic but immunostimulatory agent from the screen to improve the presentation of tumor antigens to the host immune system," said Estuardo AguilarCordova, CEO of Advantagene Inc.

Advantagene's lead compound is ProstAtak, which is being tested in combination with radiation therapy in a Phase III trial in patients with newly diagnosed prostate cancer. ProstAtak is an adenovirus vector encoding herpes simplex virus thymidine kinase (HSV-tk) coadministered with an antiherpetic drug. The therapy induces immunogenic cell death in tumor cells but the vector itself also creates a danger signal that further stimulates the immune system.

\section{Standard of care complement}

Subgroup analysis of the retrospective cancer patient data suggests Aguilar-Cordova has the right idea.

In that analysis, digoxin did not confer additional survival benefit in patients treated with chemotherapy that induced immunogenic cell death. Therefore, Kroemer thinks the cardiac glycosides are best suited for use with cancer therapies that do not induce immunogenic cell death, such as cisplatin.

"When you look at the wide use of cisplatin, you see it is quite efficient in reducing tumor mass but also inefficient in conferring any long-term benefit to the patient," Kroemer told SciBX. "Our idea is that if we could combine cisplatin with other agents to make it more immunogenic, we may be able to improve long-term patient outcomes."

The cardiac glycosides and other molecules that induce immunogenic cell death could also complement existing antigen-specific cancer vaccines, adoptive cell transfer therapies, and drugs that non-specifically stimulate T cells, such as Yervoy ipilimumab, added Pierre Coulie.

"Traditional cancer vaccine and adoptive transfer therapies generally target only a few tumor antigens," said Coulie. "Molecules that induce immunogenic cell death could give the host immune system an opportunity to react against more tumor antigens, including individual mutated antigens, and thus mount a stronger antitumor immune response."

Coulie is a professor of immunology and leader of the Human Tumor Immunology group at the de Duve Institute and the Université Catholique de Louvain. 


\section{ANALYSIS}

Bristol-Myers Squibb Co. markets Yervoy, a human mAb against CTLA-4 (CD152) receptor, to treat unresectable or metastatic melanoma.

Kroemer said his group is developing additional cancer cell lines to use with the screening platform and is trying to make the technology compatible with all cancer cell lines. In addition, the group is modifying the screening platform to identify target-specific compounds that also induce immunogenic cell death.

Aguilar-Cordova added that it is important to increase the throughput of future iterations of the screening platform.

As for the lead cardiac glycoside screening hits, Kroemer said the group plans to start enrolling patients with locally invasive head and neck cancers in an investigator-led Phase I/II trial in 2013.

"Such cancers are usually treated with cisplatin and radiotherapy, so we would combine the classical therapies with a cardiac glycoside," he told SciBX. "For the primary endpoints, we plan to measure infiltration of tumor by T cells and also therapeutic efficacy."

The Gustave Roussy Institute has filed two patent applications covering methods and compounds to induce immunogenic cell death. The work is available for licensing.

Lou, K.-J. SciBX 5(31); doi:10.1038/scibx.2012.808

Published online Aug. 9, 2012

\section{REFERENCES}

1. Menger, L. et al. Sci. Transl. Med.; published online July 18, 2012; doi:10.1126/scitranslmed.3003807

Contact: Guido Kroemer, Georges Pompidou European Hospital, Paris, France

e-mail: kroemer@orange.fr

Contact: Oliver Kepp, University of South Paris XI, Bicêtre, France e-mail: oliver.kepp@igr.fr

2. Casares, N. et al. J. Exp. Med. 202, 1691-1701 (2005)

3. Obeid, M. et al. Nat. Med. 13, 54-61 (2007)

4. Panaretakis, T. et al. Cell Death Differ. 15, 1499-1509 (2008)

5. Ghiringhelli, F. et. al. Nat. Med. 15, 1170-1178 (2009)

6. Apetoh, L. et al. Nat. Med. 13, 1050-1059 (2007)

7. Prassas, I. \& Diamandis, E.P. Nat. Rev. Drug Discov. 7, 926-935 (2008)

\section{COMPANIES AND INSTITUTIONS MENTIONED}

Advantagene Inc., Auburndale, Mass. Bristol-Myers Squibb Co. (NYSE:BMY), New York, N.Y. de Duve Institute, Brussels, Belgium Georges Pompidou European Hospital, Paris, France Gustave Roussy Institute, Villejuif, France Institut National de la Santé et de la Recherche Médicale, Villejuif, France

Université Catholique de Louvain, Brussels, Belgium

University of Navarra, Pamplona, Spain

University Paris Descartes, Paris, France 